\title{
Whole-Cell Tomography Using a Conventional Scanning Electron Microscope
}

Taiga Okumura ${ }^{1}$, Minami Shoji ${ }^{1}$, Akiko Hisada ${ }^{1}$, Yusuke Ominami ${ }^{2}$, Tatsuo Ushiki ${ }^{3}$, Masato Nakajima ${ }^{3}$ and Takashi Ohshima ${ }^{1}$

1. Hitachi, Ltd., Central Research Laboratory, Tokyo, Japan

2. Hitachi High-Technologies Corporation, Ibaraki, Japan

3. Graduate School of Medical and Dental Sciences, Niigata University, Niigata, Japan

Traditionally, ultrastructure of biological samples has been elucidated predominantly using transmission electron microscopy (TEM). For TEM observation, the samples are embedded in resin and cut into ultrathin sections using an ultramicrotome. These sample preparation procedures, however, require skills to make adequate electron-transparent thin sections on a small grid, which is obstacle to TEM examination. To overcome the difficulty, we developed plate-transmission electron microscopy (PlateTEM), by which internal structure of biological samples is observed using a transmitted electron (TE) mode of scanning electron microscopy (SEM) with a scintillator plate [1,2]. Furthermore, whole-cell tomography was conducted using this technique in order to image the three-dimensional (3D) structure of the samples.

In this technique, the samples on a transparent scintillator plate are irradiated with an incident electron beam, and the plate emits scintillation light (photons) produced by electrons that pass through the samples (Fig. 1). Some of the electrons are backscattered and absorbed by dense materials inside the samples, which means that the quantity of penetrating electrons and emitted photons varies with the density of the samples. Thus Plate-TEM achieves TE imaging without the sample-sectioning process. In addition, the sample surface can be observed simultaneously by detecting secondary and/or backscattered electrons (SEs/BSEs).

Plate-TEM cannot directly reveal the 3D structure because the compositions of biological samples overlap with each other in TE images. Therefore we made a new sample stage to tilt the samples for Plate-TEM tomography. The new tilt-stage was fabricated by installing a small motor on a sample stage and attaching a scintillator plate to the motor shaft. Photons are emitted from the underside of the plate and detected by a photodetector. A tilt-series $\left( \pm 60^{\circ}\right.$ with an increment angle of approximately $\left.2^{\circ}\right)$ was acquired using a conventional SEM. Image alignment and 3D reconstruction were carried out with IMOD [3], and image visualization was performed with Avizo (Mercury Computer Systems).

Indian muntjac thymus cells (Mm2T) were examined using the Plate-TEM tomography. The cells were cultivated on the scintillator plates, fixed in glutaraldehyde, postfixed in osmium tetroxide, dehydrated in an ethanol series, critical point-dried, and coated with carbon to avoid specimen charging. Figure 2A and $2 \mathrm{~B}$ shows the SE and Plate-TEM images of the same cell, respectively. The cell surface was observed in the SE image, whereas several organelles such as mitochondria were recognized in the Plate-TEM image. Furthermore, the tomography with Plate-TEM images generated the volume-rendered 3D model (Fig. 3A) and the slice image (Fig. 3B). Thus this technique enables us to inspect 3D distribution and forms of organelles from various viewing angles.

In conclusion, Plate-TEM tomography is a valuable technique for exploring 3D internal structure of biological samples using a conventional SEM without destructive sample-sectioning processes. 
References:

[1] Y Ominami et al, Scanning Microscopies (2014) 923608.

[2] Y Ominami et al, Microscopy and Microanalysis (2014) 32-33.

[3] JR Kremer, DN Mastronarde and JR McIntosh, J. Struct. Biol. 116 (1996) 71-76.

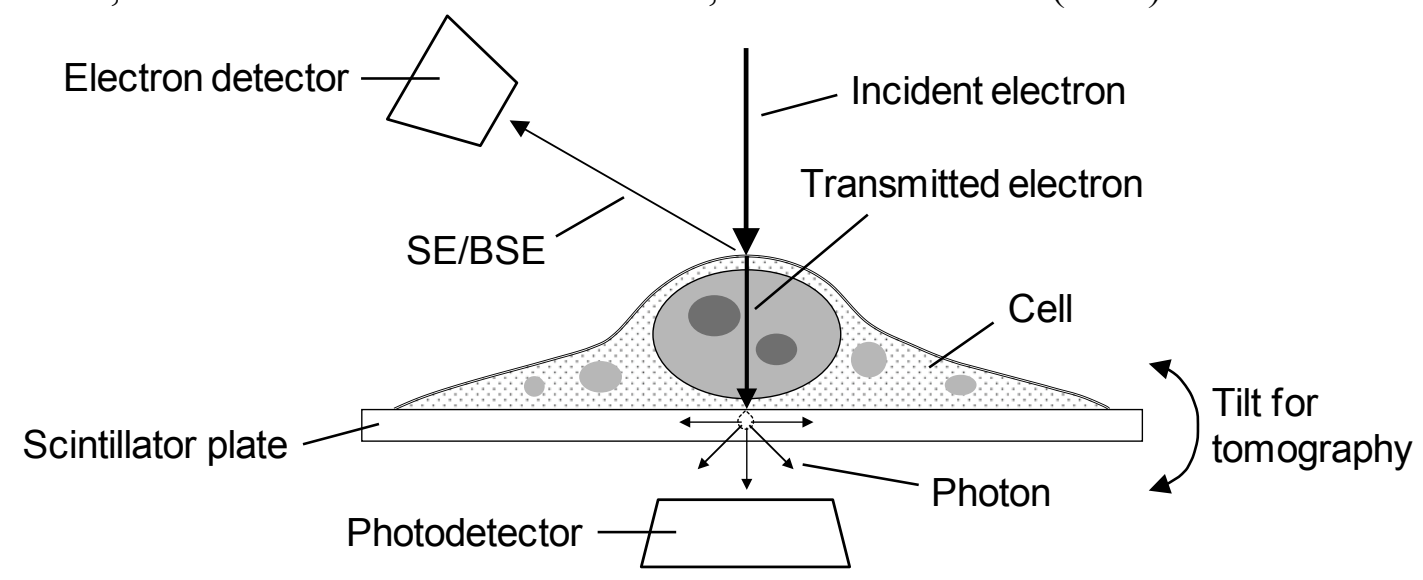

Figure 1. Schematic of Plate-TEM.
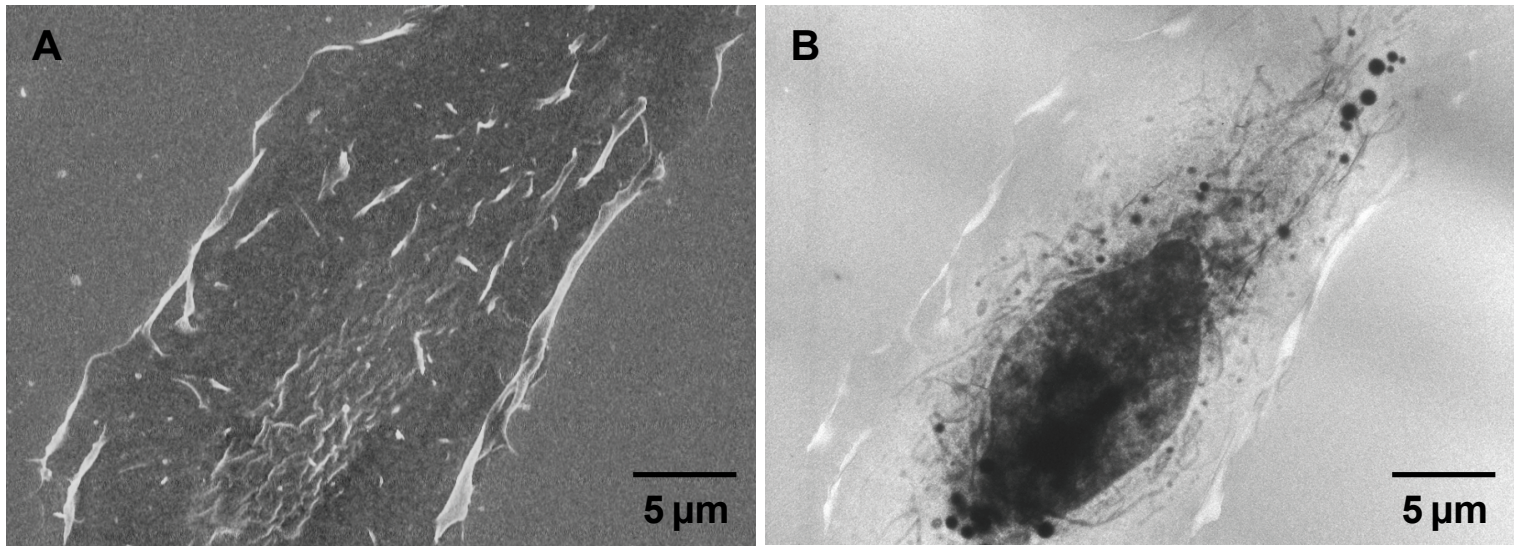

Figure 2. SE (A) and Plate-TEM (B) images of the Mm2T cell.
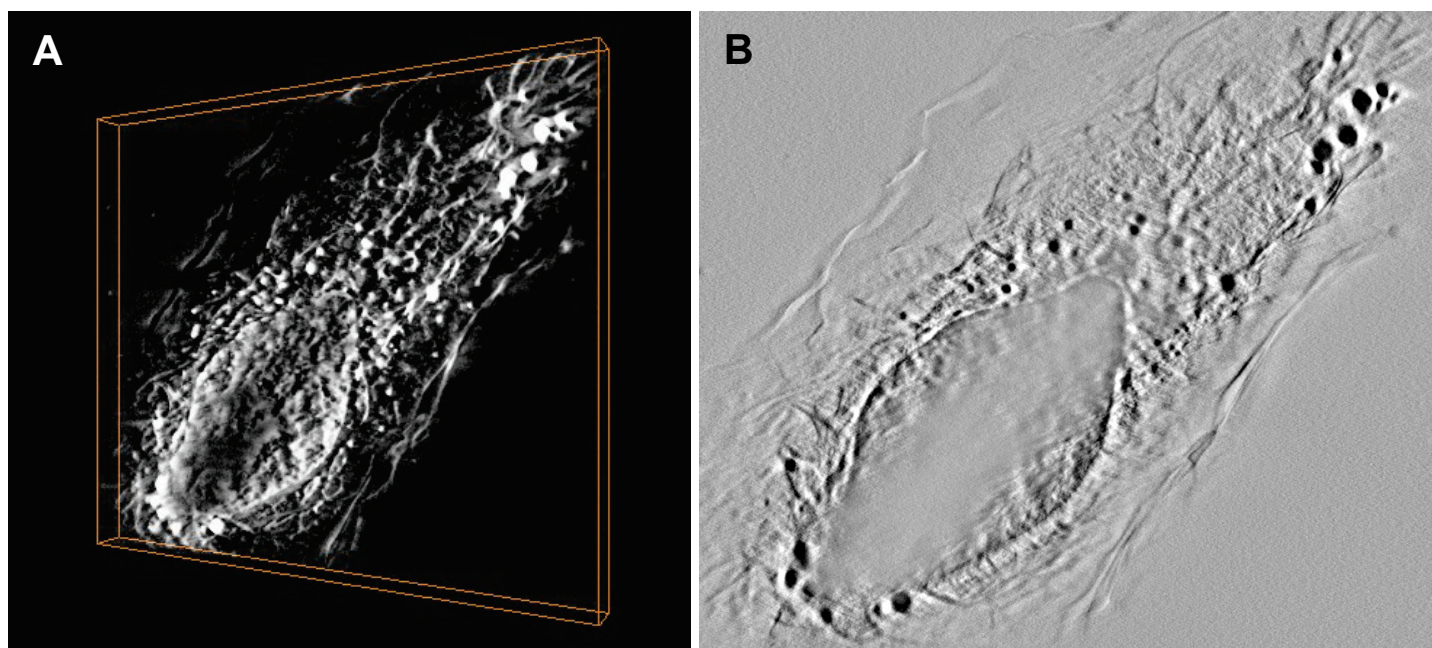

Figure 3. Volume-rendered 3D model (A) and Slice image (B) of the Mm2T cell. 